NUMEHICAL ANALYSIS AND MATHEMATICAL MODELLING BANACH CENTER PUDLIC.ATIONS, VOLUME 24 PWN-POLISH SCIENTIFIC PUBLISHERS

WARSAW 1990

\title{
ВАРИАЦИОННО-РАЗНОСТНЫЕ МЕТОДЫ РЕШЕНИЯ ПАРАБОЛИЧЕСКИХ УРАВНЕНИЙ
}

\author{
ю. Р. АКОПЯН \\ Факульпет приклаdной математаки, \\ Ереванский государстоенивй университет, \\ Epesarı, СССР
}

В настоящей работе обсуждаются вопросы построения предельных по порядку в смысле поперечников вариационно-разностных схем (ВРС) для двумерных линейных параболических уравнений с правой частью из пространства $L_{2}$.

\section{$\S 1$. Постановіса шачально-краевых задач}

1. Рассмотрим в цилиндрической области $Q=\Omega \times(0, T)$, где $\Omega$ - ограниченная односвязная область с границей $S$ в плоскости $\boldsymbol{R}_{2}$ точек $x=\left(x_{1}, x_{2}\right)$, уравнение

$$
\frac{\partial u}{\partial t}-\sum_{i, j=1}^{2} \frac{\partial}{\partial x_{i}}\left(a_{i j} \frac{\partial u}{\partial x_{j}}\right)+a u=f
$$

с начальным условием

$$
u(x, 0)=0, \quad x \in \Omega
$$

и краевым условием одного из двух типов:

$$
u(x, t)=0, \quad(x, t) \in S \times(0, T)
$$

или

$$
\frac{\partial u}{\partial N}+\sigma u=0, \quad(x, l) \in S \times(0, T),
$$

где

$$
\frac{\partial u}{\partial N} \equiv \sum_{i, j=1}^{2} a_{i j} \frac{\partial u}{\partial x_{j}} \cos \left(v, x_{i}\right)
$$

здесь $v-$ внешняя нормаль к кривой $S$. 
Относительно уравнения (1) предполагается, что $a_{i j}=a_{i j}, i, j=1,2$, и существует такая положительная постоянная $\gamma$, чго неравенство

$$
\sum_{i, j=1}^{2} a_{i j}(x, t) \xi_{i} \xi_{i} \geqslant \gamma \sum_{i=1}^{2} \xi_{i}^{2}
$$

выполняется для всех $(x, t) \in Q$ и $\xi=\left(\xi_{1}, \xi_{2}\right) \in \boldsymbol{R}_{2}$.

Задача (1), (2), (3), - первая начально-краевая задача.

Задача (1), (2), (4), - третья начально-краевал задача.

2. В работе приняты следующие обозначения для норм в функциональных пространствах:

$\|\cdot\|_{0, \Omega}$ - норма в пространстве $L_{2}(\Omega)$;

$\|\cdot\|_{k, \Omega}$ - норма в пространстве Соболева $W_{2}^{k}(\Omega)$;

$\|\cdot\|_{0, Q}$ - норма н пространстве $L_{2}(Q)$;

$\|\cdot\|_{k, m, Q}$ - норма в пространстве Соболева-Слободецкого $W_{2}^{k, m}(Q)$.

$V_{2}^{1,0}(Q)$ - банахово пџространство функций с нормой

$$
|u|_{Q} \equiv \sup _{0 \leqslant t \leqslant T}\|u(t)\|_{0, \Omega}+\|\mid \nabla u\|_{0, Q},
$$

где

$$
|\nabla u| \equiv\left(\sum_{i=1}^{2}\left(\frac{\partial u}{\partial x_{i}}\right)^{2}\right)^{1 / 2}
$$

3. Предположим, что граница $S$ есть глацкая кривая класса $C^{2}$, а правая часть $f$ уравнения (1) принадлежит пространству $L_{2}(Q)$. При определенных условиях, накладываемых на гладкость коэффициентов уравнения (1) и функцию $\sigma$ из (4), существует единственное решение как первой, так и третьей начально-краевой задачи из пространства $W_{2}^{2,1}(Q)$ и справедливо неравенство

$$
\|u\|_{2,1, Q} \leqslant C_{1}\|f\|_{0, Q}\left({ }^{1}\right)
$$

(см., напр., работы [1], [2]).

\section{§ 2. Неявная ВРС для третьей начальюо-красвой задачи}

1. Наложим на область $\Omega$ квадратную сетку шага $h$, обра́зованную линиями, паралыельными осям координат. Каждую ячейку квадратной сетки разобьем диагональю под углом $45^{\circ}$ к оси $x_{1}$ на два треугольника. Наименьшее объединение треугольников, содержашее $\Omega$, обозначим через $\Omega_{e x}^{h}$ и назовем сеточной областью. Совокупность вернин и сторон

(1) В далнейшем буквой С с индексами внизу обоэначаются различные положительные постоянные в неравенствах, пе зависяцие от рядом стоянцх множигелей и шага сетки. 
трегугольников, из которых состоит сеточная область $\Omega_{e x}^{h}$, образует регулярную сетку. Вершины треугольников назовем узлами сетки. Множество всех узлов обозначим через $\overline{R^{\prime \prime}}$.

Будем считать, что все узлы сетки перенумерованы в некотором порядке и $x^{k}=\left(x_{1}^{k}, x_{2}^{k}\right)$ есть $k$-й узел сетки. Для произвольной сеточной функции

$$
v=\left\{v_{k} \equiv v\left(x^{k}\right) \mid x^{k} \in \overline{R^{h}}\right\}
$$

определим ее кусочно-линейное восполнение

$$
\tilde{v}(x)=\sum_{x^{k} \in \bar{R}^{h}} v_{k} \varphi_{k}(x),
$$

где $\varphi_{k}(x)$ есть базисная функция кусочно-линейного восполнения, соответствующая узлу $x^{k}$. Конечномерное пространство функций вида (5) обозначим через $H_{h}$. Ясно, что $H_{h} \subset W_{2}^{1}\left(\Omega_{e x}^{h}\right)$.

Теперь перейдем к восполнениям в трехмерном пространстве. Возьмем на отрезке $[0, T]$ равноотстоящие точки $t_{n}=n \tau, n=0,1, \ldots, N$, с шагом $\tau ; t_{N}=T$. Определим базисные функции

$$
\varphi_{k}^{\prime \prime}(x, t)=\left\{\begin{array}{cc}
\varphi_{k}(x), & \text { если } t \in\left(t_{n-1}, t_{n}\right], \\
0, & \text { если } t \notin\left(t_{n-1}, t_{n}\right]
\end{array}\right.
$$

для $n=1,2, \ldots, N$.

Прибдиженное решение третьей начально-караеной задачи будем искать в конечномерном пространстве $H_{h}^{\tau}$ функций вида

$$
\tilde{\tilde{v}}(x, t)=\sum_{n=1}^{N} \sum_{x^{k} \in R^{n}} v_{k}^{\prime \prime} \varphi_{k}^{n}(x, t) .
$$

Функции из $H_{h}^{\tau}$ являются кусочно-линейными по пространственным переменным и кусочно-постоянньми по времени. Для каждого $t \in\left(t_{n-1}, t_{n}\right]$

$$
\tilde{v}(x, t) \equiv \tilde{v}\left(x, t_{n}\right) \equiv \tilde{v}^{n}(x),
$$

где

$$
\tilde{v}^{n}(x)=\sum_{x^{k} \in R^{n}} v_{k}^{n} \varphi_{k}(x) \in H_{n} .
$$

2. Приближенным решением третьей начально-краевой задачи назовем функцию $\tilde{\tilde{v}} \in H_{h}^{\tau}$, удовлетворяющую интегральному тождеству

$$
\sum_{n=1}^{N} \int_{\Omega}\left(\tilde{v}^{n}-\tilde{v}^{n-1}\right) \tilde{\varphi}^{n} d x+B(\tilde{\tilde{v}}, \tilde{\tilde{\varphi}})=\int_{0}^{T} \int_{\Omega} f \tilde{\tilde{\varphi}} d x d t
$$

при произвольной $\tilde{\tilde{\rho}} \in H_{h}^{\tau}$, где билинейная форма $B$ определяется следуюшим образом:

$$
B(u, v) \equiv \int_{0}^{T} \int_{\Omega}\left[\sum_{i, j=1}^{2} a_{i j} \frac{\partial u}{\partial x_{j}} \frac{\partial v}{\partial x_{i}}+a u v\right] d x d t+\int_{0}^{T} \int_{S} \sigma u v d s d t
$$


В склу произвольности функции $\tilde{\tilde{\varphi}}$ из (6) следует, что для любого $n=1,2, \ldots, N$

$$
\int_{\Omega}\left(\tilde{v}^{n}-\tilde{v}^{n-1}\right) \tilde{\varphi} d x+B_{n}\left(\tilde{v}^{n}, \tilde{\varphi}\right)=\int_{l_{n-1}}^{t_{n}} \int_{\Omega} f \tilde{\varphi} d x d t
$$

где $\tilde{\varphi}$ есть произвольная функция из $H_{b}$; а̇

$$
B_{n}\left(\tilde{v}^{n}, \tilde{\varphi}\right) \equiv \int_{t_{n-1}}^{t_{n}} \int_{\Omega}\left[\sum_{i, j=1}^{2} a_{i j} \frac{\partial \tilde{v}^{n}}{\partial x_{j}} \frac{\partial \tilde{\varphi}}{\partial x_{i}}+a \tilde{v}^{n} \tilde{\varphi}\right] d x d t+\int_{n_{n}-1}^{t_{n}} \int_{S} \sigma \tilde{v}^{n} \tilde{\varphi} d s d t .
$$

Таким образом, из (7) получим, что для нахождения приближенного решения на каждом слое $n=1,2, \ldots, N$ следует решать систему сеточных уравнений

$$
\left(P+L_{n}\right) v^{n} .=P v^{n-1}+r^{n},
$$

где матрицы $P, L_{n}$ и вектор $r^{n}$ таковы, что

$$
\begin{gathered}
\int_{\Omega} \tilde{w} \tilde{\varphi} d x=(P w, \varphi), \quad B_{n}(\tilde{w}, \tilde{\varphi})=\left(L_{n} w, \varphi\right) \\
\int_{t_{n-1}}^{t_{n}} \int_{\Omega} f \tilde{\varphi} d x d t=\left(r^{n}, \varphi\right)
\end{gathered}
$$

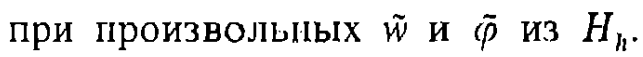

При достаточно малом $\tau$ системы (8) однозначно разрешимы.

3. В дальнейшем предполагается, что $\tau=O\left(h^{2}\right)$. Ниже, в $\S 4$, будет обоснован такой выбор соотношения между шагами сетки.

В работах [3], [4], [5] получешы следуюпџе оценки скорости сходимости построеннгй ВРС:

$$
\begin{aligned}
|u-\tilde{\tilde{v}}|_{Q} & \leqslant C_{2} h\left\|f^{\prime}\right\|_{0, Q}, \\
\|u-\tilde{\tilde{v}}\|_{0, Q} & \leqslant C_{3} h^{2}\|f\|_{0, Q} .
\end{aligned}
$$

Эти оценки, как будет показано в $\$ 4$, можно трактовать в некотором смысые как пределыные.

Вопросам исследования скорости сходимости неянных ВРС 'для параболических уравнений с правой частью из прострапства $L_{2}$ посвяпены также работы [6], [7].

4. Будем решать системы сеточных уравнений (8) na n-ом слое итерационным методом

$$
\begin{aligned}
& D \frac{v^{n}(k+1)-v^{n}(k)}{\lambda}=-\left(P+L_{n}\right) v^{n}(k)+P v^{n-1}(q)+r^{n}, \\
& k=0,1,2, \ldots ; \quad v^{n}(0)=v^{n-1}(q),
\end{aligned}
$$


где $\lambda$ - положительный параметр, а $D=\operatorname{diag}\left\{d_{1}, d_{2}, \ldots, d_{M}\right\}$ диагональная матрица ( $M$ - число узлов в множестве $\left.\overline{R^{h}}\right)$. Диагональные элементы матрицы $D$ выбираются следующим образом [8]. Как уже отмечалось выше, триангуляция плоскости осуцествляется пугем разбиения ячеек квадратной сетки диагональю на два треугольника. Обозначим через $\Delta_{j}^{(k)}, j=1,2, \ldots, 6$, треугольники, имеюшие узел $x^{k}=\left(x_{1}^{k}, x_{2}^{k}\right)$ своей общей вершиной. При этом треугольники занумерованы в направлении против часовой стрелки, начиная с треутольника $\Delta_{1}^{(k)}$ с вершинами $\left(x_{1}^{k}, x_{2}^{k}\right)$, $\left(x_{1}^{k}+h, x_{2}^{k}\right)$ и $\left(x_{1}^{k}+h, x_{2}^{k}+h\right)$. Тогда

$$
d_{k}=\frac{1}{4} \sum_{j=1}^{6} \operatorname{mes}\left(\delta_{j}^{(k)}\right)+\frac{1}{4} \operatorname{mes}\left(\delta_{3}^{(k)} \cup \delta_{6}^{(k)}\right),
$$

где $\delta_{j}^{(k)}=\Delta_{j}^{(k)} \cap \Omega$. Заметим, что для „внутренних" узлов (т.е. для которых $\delta_{j}^{(k)}$ совпадают с $\Delta_{j}^{(k)}$ при всех $\left.j=1,2, \ldots, 6\right) d_{k}=h^{2}$.

В работе [5] сформулирована и доказана теорема о сходимости итерационного метода (II). Утверждается, что параметр $\lambda=$ const можно выбрать так, что совершая на каждом слое $q=O\left(\ln h^{-1}\right)$ итерацнй, можно получить приближенное решление с точностью, даваемой оценками (9) и (10). Отметим при этом, что

$$
\operatorname{cond}\left(D^{-1 / 2}\left(P+L_{n}\right) D^{-1 / 2}\right)=O(1) .
$$

\section{§ 3. Явная ВРС для первой началыю-краевой задачи}

1. Построим для области $\Omega$ сеточную область $\Omega_{i n}^{\prime \prime} \subset \Omega$, ограниченную ломаной $S^{h}$ и удовлетворяющую следующим свойствам:

(а) между точками $S^{h}$ и $S$ с помоцью нормалей к $S$ устанавливается взаимно-однозначное соответствие;

(б) рассгояния точек $S^{h}$ до $S$ не превосходят величины $\delta_{1} h^{2}$, где $\delta_{2}>0$ не зависит от $h$;

(в) область $\Omega_{l n}^{h}$ триангулирована таким образом, что треугольники триангуляци имеют длины сторон, лежащие в пределах $\left[\delta_{2} h ; \delta_{3} h\right]$, а плошади треугольников лежат в пределах $\left[\delta_{4} h^{2} ; \delta_{5} h^{2}\right]$, где постоянные $\dot{\delta}_{3} \geqslant \dot{\delta}_{2}>0, \dot{\delta}_{5} \geqslant \dot{\delta}_{4}>0$ не занислт от $h$.

(г) наa расстоянии порядка $h$ от границы области триангуляция регулярная.

Существуют алгоритмы автоматического построения нерегулярной сетки, удовлетворяюцие перечисленным свойствам [9].

Обозначим через $R^{h}$ множество внутренних (т.е. не принадлежаших $S^{h}$ ) узлов сетки. Дляя произвольной сеточной функции

$$
v=\left\{v_{k} \equiv v\left(x^{k}\right) \mid x^{k} \in R^{h}\right\}
$$


определим кусочно-линейное восполнение

$$
\hat{v}(x)=\sum_{x^{k} \in R^{\prime \prime}} v_{k} \varphi_{k}(x) .
$$

Базисные функции $\varphi_{k}(x)$ считаются продолженными нулем на полосу $\Omega \backslash \Omega_{\text {in. }}^{h}$. Конечномерное пространство функций вида (12) обозначим через

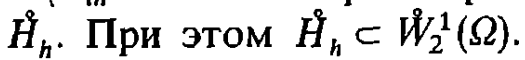

Пусть $t_{n}=n \tau, n=0,1,2, \ldots, N ; t_{N}=T$.

Определим билинейную форму

$$
B_{n}(u, v) \equiv \int_{l_{n}}^{t_{n+1}} \int_{\Omega}\left[\sum_{i, j=1}^{2} a_{i j} \frac{\partial u}{\partial x_{j}} \frac{\partial v}{\partial x_{l}}+a u v\right] d x d t
$$

для $n=0,1, \ldots, N-1$.

Для решения задачи запишем следуюшую схему

$$
h^{2}\left(p^{n+1}-v^{n}\right)+L_{n} v^{n}=g^{n}, \quad n=0,1, \ldots, N-1 ; v^{0}=0,
$$

где матрица $L_{n}$ и вектор $g^{n}$ таковы, что

$$
B_{n}(\hat{v}, \hat{w})=\left(L_{n} v, w\right), \quad \int_{l_{n}}^{t_{n+1}} \int_{\Omega} f \hat{w} d x d t=\left(g^{n}, w\right)
$$

для произвольных $\hat{w}$ и $\hat{\varphi}$ из $H_{h}$.

Схема (13) позволяет вычислять на каждом слое сеточную функцию $v^{n}$ и ее кусочно-линейное восполнение $\hat{v}^{n}(x)$. Далее, определяется функция

$$
\hat{\hat{v}}(x t)= \begin{cases}\hat{v}^{n}(x) & \text { если } t \in\left[t_{n}, t_{n+1}\right), n=0,1, \ldots, N-1, \\ \hat{v}^{N}(x), & \text { если } t=t_{N}\end{cases}
$$

которую будем считать приближенным решением первой начально-краевой задачи.

2. В работах [10], [11], в предположении, что $\tau=O\left(h^{2}\right)$, получены следующие оценки скорости сходимости

$$
\begin{aligned}
|u-\hat{v}|_{Q} & \leqslant C_{4} h\|f\|_{0, Q}, \\
\|u-\hat{v}\|_{0, Q} & \leqslant C_{5} h^{2}\|f\|_{0, Q} .
\end{aligned}
$$

\section{§ 4. О точности получеших оценок}

Полученные нами выше оценки скорости сходимости ВРС (9), (10), (14) и (15), при условии, что $\tau=O\left(h^{2}\right)$, имеют вид

$$
\begin{gathered}
|u-v|_{Q} \leqslant C_{6} h\|f\|_{0, Q}, \\
\|u-v\|_{0, Q} \leqslant C_{7} h^{2}\|f\|_{0, Q},
\end{gathered}
$$

( $u$ - точное решение, $v-$ приближенное решение). 
Запишем эти оценки в несколько ином виде. Обозначим через $M$ число узлов сетки, построенной для области $\Omega$; очевидно, что $M=O\left(h^{-2}\right)$. Приближенное решение мы искали как элемент конечномерного пространства функций, порожденного $M N$ базисными функциями, где $N=O\left(\tau^{-1}\right)$. Пусть $R=M N$. Тогда оценки (16) и (17) примут вид:

$$
\begin{aligned}
|u-v|_{Q} & \leqslant C_{8} R^{-1 / 4}\|f\|_{0, Q}, \\
\|u-v\|_{0, Q} & \leqslant C_{9} R^{-1 / 2}\|f\|_{0, Q} .
\end{aligned}
$$

Зададимся вопросом: точны ли по порядку наши оценки и нельзя ли их улучшить за счет иного выбора того же, или меньшего, числа базисных функций. Для этого воспользуемся понятием поперечника по А. Н. Колмогорову.

Будем рассматривать приближенные методы, состоящие в том, что приближенное решение начально-краевой задачи при правой части $f$ из $L_{2}(Q)$ ишется как элемент $R$-мерного подпространства $L_{R}$.

Пусть $K$ - множество решений начально-краевой задачи, соответствующих всевозможным правым частям $f$ уравнения (1) из шара $\|f\|_{0, Q} \leqslant 1$. Определим $R$-поперечник

$$
\begin{aligned}
& d_{R}(K)=\inf _{L_{R} \subset V_{2}^{1,0}(Q)} \sup _{u \in K} \inf |u-v|_{v \in L_{R}}, \\
& d_{R}^{*}(K)=\inf _{L_{R} \subset L_{2}(Q)} \sup _{u \in K} \inf \|u-v\|_{0, Q} .
\end{aligned}
$$

Оценки сверху для обоих поперечников

$$
d_{R}(K) \leqslant C_{8} R^{-1 / 4}, \quad d_{R}^{*}(K) \leqslant C_{9} R^{-1 / 2}
$$

фактически следуют из оценок (18) и (19). С другой стороны, в работе [3] доказывается, что

$$
d_{R}(K) \leqslant C_{10} R^{-1 / 4}, \quad d_{R}^{*}(K) \leqslant C_{11} R^{-1 / 2} .
$$

Поэтому полученные оценки скорости сходимости ВРС являются точными по порядку в смысле поперечников.

\section{Литература}

[1] О.А. Ладыженская, В. А. Солонников и Н. Н. Уральцева, Липйные и квазилииейнbе уравиения параболического типа, Наука, Москва 1967.

[2] А. М. Ильнн, А. С. Калашников и О. А. Олейник, Линейлье уравнения второго порядка параболического тина, Успехи математических наук 17 вып. 3 (105) 1962, 3-146.

[3] Ю. Р. Акопян и Л. А. Оганесян, Вариационо-разиосишый метод ремения двумериых лимейых параб̆олческих уравнений, Журнал вычислит. матем. и матем. физики 17 (1) $1977,109-118$. 


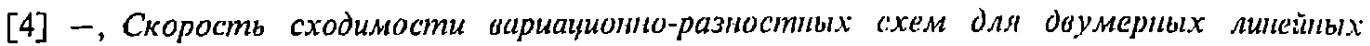
параболических уравнений, В сб.: Вариационно-разностные методы решения задач математической фнзики, ВЦ СО АН СССР, Новосибирск 1976, 27-36.

[5] Ю. Р. Акопян,_ккорость сходиности в $L_{2}$ вариационо-разиостиых схем дм даумерных липейиых параболических уравпений. Иперациониое ремение раэностиых уравнений, Доклады АН Арм. ССР 62 (4) 1976, 193-198.

[6] А. А. Злотник, Оченка скорости сходимости в $L_{2}$ проскииопио-разиостиых схем дая параболических ураншенй, Журнал вычислит. матем. и матем. физики 18, (6) 1978, $1454-1465$.

[7] -, О скорости сходиности проекционио-разностиых схем оля параболических уравнелий, В сб.: Вариационно-разностные методы в математичсской физике, часть 1, М., OBM АН CCCP 7280.

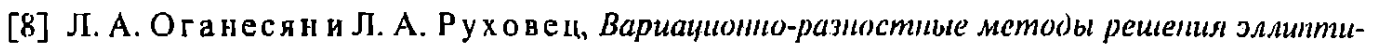
ческих ураянений, Ереван 1979.

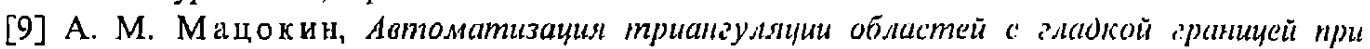
рециении уравиений эллиптического muna, Препринт № 15 семинара Вычнслительные мстоды прикладной математики, ВЦ СО АН СССР, Новосибирск 1975.

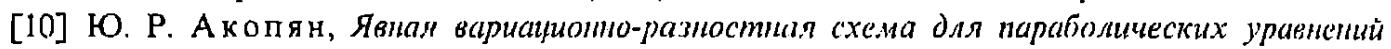
с правой частыо из $L_{2}$, В сб.: Прикладная математика, вып. 2, Еревян 1983, 5-19.

[11] -, Оуенка скорости сходимости а $L_{2}$ яоной нариауноно-разиостиой схемы для параболических уравиений, В сб.: Вариационно-разностные мстоды в математической физике, часть I, М., ОВМ АН С.ССР 1984, 12-25.

Presented to the Semester

Numerical Analysis and Mathematical Modelling

February 25-May 29, 1987 